\title{
Reduced Order Finite Time Observers for Time-Varying Nonlinear Systems
}

\author{
Frederic Mazenc \\ Saeed Ahmed \\ Michael Malisoff
}

\begin{abstract}
We build reduced order finite time observers for a large class of nonlinear time-varying continuous-time systems. We illustrate our results using a tracking problem for nonholonomic systems in chained form.
\end{abstract}

Index Terms-Observer, stability, time-varying

\section{INTRODUCTION}

The problem of estimating the values of solutions of systems when some variables cannot be measured is of great relevance from the theoretical and applied points of view. Asymptotic observers, such as the celebrated Luenberger observer from [5] and [6], are very popular and many observers for families of nonlinear systems have been constructed. However, they usually only provide a useful estimate after a transient period during which they cannot be used, which can be a disadvantage in applications like fault detection where a finite time state estimation is desirable [12].

To obtain an exact estimate of the solutions of a system in an arbitrarily short amount of time, finite time observers have been proposed. Some use nonsmooth functions; see for instance [4] and [11]. Their designs are often based on homogeneous properties that preclude the possibility of deriving smooth observers from this technique. Another type of finite time observers has been developed. They are smooth and use past values of the output or dynamic extensions. They have been proposed a few decades ago for linear systems; see in particular [2] and [13]. More recently, finite time observers were designed for nonlinear systems, e.g., in [9], [10], and [15]. They apply to systems whose vector field is time-invariant when the output is set to zero and provide estimates of all of the state variables.

Since systems are frequently time-varying, and since tracking problems can be recast into stabilization problems for equilibria of time-varying systems, and since the measured components of the state do not need to be estimated, this paper adapts the main results of [9] and [15] to construct finite time reduced order observers for a family of nonlinear time-varying systems. The observers we will build only give estimates of the unmeasured variables, as do the asymptotic observers proposed for instance in [3] and [1, Chapt. 4,

Mazenc is with EPI DISCO INRIA-Saclay, Laboratoire des Signaux et Systèmes (L2S, UMR CNRS 8506), CNRS, CentraleSupélec, Université Paris-Sud, 3 rue Joliot Curie, 91192, Gif-sur-Yvette, France, frederic.mazenc@12s.centralesupelec.fr.

Ahmed is with Department of Electrical and Electronics Engineering, Graduate School of Engineering and Science, Bilkent University, Ankara 06800, Turkey, ahmed@ee.bilkent.edu.tr.

Malisoff is with the Department of Mathematics, Louisiana State University, Baton Rouge, LA 70803-4918, USA, malisoff@1su.edu.

Malisoff was supported by NSF Grant 1711299.
Sec. 4.4.3]. This provides a technical advantage over other observers that would require fundamental solutions of timevarying linear systems whose dimensions are equal to those of the original systems. This is because we only need formulas for fundamental solutions of lower order time-varying systems, and because of the difficulty of finding formulas for fundamental solutions of higher dimensional time-varying linear systems. To the best of our knowledge, finite time reduced order observers for nonlinear time-varying systems are proposed for the first time in the present paper.

After providing an introductory result in Section II, we state and prove a reduced order finite time observer theorem for time-varying nonlinear systems in Section III. Section IV illustrates our approach, in a tracking problem for a nonholonomic system in chained form, and we conclude with ideas for future research in Section V.

We use standard notation, which is simplified whenever no confusion can arise, and the dimensions of our Euclidean spaces are arbitrary, unless otherwise noted. The Euclidean norm, and the induced norm of matrices, are denoted by $|\cdot|,|\cdot|_{\infty}$ is the essential supremum, and $I$ is the identity matrix. For each constant $\tau>0$, continuous function $\varphi$ : $[-\tau,+\infty) \rightarrow \mathbb{R}^{n}$, and $t \geq 0$, we define $\varphi_{t}$ by $\varphi_{t}(m)=$ $\varphi(t+m)$ for all $m \in[-\tau, 0]$. For any continuous function $\Omega:[-\tau,+\infty) \rightarrow \mathbb{R}^{n \times n}$, we let $\Phi_{\Omega}$ denote the function such that

$$
\frac{\partial \Phi_{\Omega}}{\partial t}\left(t, t_{0}\right)=-\Phi_{\Omega}\left(t, t_{0}\right) \Omega(t)
$$

and $\Phi_{\Omega}\left(t_{0}, t_{0}\right)=I$ for all $t \in \mathbb{R}$ and $t_{0} \in \mathbb{R}$. Then $\mathcal{M}(t, s)=\Phi_{\Omega}^{-1}(t, s)$ is the fundamental solution associated to $\Omega$ for the system $\dot{x}=\Omega(t) x$; see [16, Lemma C.4.1].

\section{INTRODUCTORY RESULT}

Before providing our general theorem for time-varying systems with nonlinearities in Section III, we first present an introductory result that builds finite time reduced order observers for a simpler family of systems, to present basic ideas from our method. Consider the system

$$
\dot{x}(t)=A x(t)+\delta(t)
$$

having $x$ valued in $\mathbb{R}^{n}$, in which $A$ is a constant matrix and the piecewise continuous function $\delta:[0,+\infty) \rightarrow \mathbb{R}^{n}$ is locally bounded. We assume that the output

$$
y(t)=C x(t)
$$

is valued in $\mathbb{R}^{p}$ and that $C$ is of full rank, where $p<n$ is arbitrary, and that $(A, C)$ is an observable pair. Since $C$ is of full rank, we can construct matrices $C_{T}$ and $A_{1}$ and $A_{2}$ 
that provide a linear change of coordinates

$$
x_{T}=C_{T} x=\left[\begin{array}{r}
y \\
x_{r}
\end{array}\right]
$$

and functions $\delta_{i}$ for $i=1,2$ that are piecewise continuous with respect to their first argument and linear with respect to $y$ for which the $x_{T}$ system can be rewritten as

$$
\left\{\begin{array}{r}
\dot{y}(t)=A_{1} x_{r}(t)+\delta_{1}(t, y(t)) \\
\dot{x}_{r}(t)=A_{2} x_{r}(t)+\delta_{2}(t, y(t)) .
\end{array}\right.
$$

Then the pair $\left(A_{2}, A_{1}\right)$ is observable, because $(A, C)$ is observable; see [6, pp. 304-306]. Since $\left(A_{2}, A_{1}\right)$ is observable, it follows from [9, Lemma 1] that there exist a constant $\tau>0$ and a constant matrix $L \in \mathbb{R}^{(n-p) \times p}$ such that with the choice $H=A_{2}+L A_{1}$, the matrix

$$
M_{\tau}=e^{-A_{2} \tau}-e^{-H \tau}
$$

is invertible.

In terms of the variable

$$
x_{s}=x_{r}+L y
$$

we easily obtain the system

$$
\dot{x}_{s}(t)=\left(A_{2}+L A_{1}\right) x_{r}(t)+\delta_{2}(t, y(t))+L \delta_{1}(t, y(t)) \text {. }
$$

Then the definition of $H$ gives

$$
\dot{x}_{s}(t)=H x_{s}(t)+K y(t)+\delta_{3}(t, y(t))
$$

where $K=-\left(A_{2}+L A_{1}\right) L$ and $\delta_{3}=\delta_{2}+L \delta_{1}$. We now integrate the second equation in (5) and (9) to obtain

$$
\begin{aligned}
& \quad x_{r}(t-\tau)=e^{-A_{2} \tau} x_{r}(t) \\
& \quad-\int_{t-\tau}^{t} e^{A_{2}(t-m-\tau)} \delta_{2}(m, y(m)) \mathrm{d} m \text { and } \\
& x_{s}(t-\tau)=e^{-H \tau} x_{s}(t) \\
& -\int_{t-\tau}^{t} e^{H(t-m-\tau)}\left[K y(m)+\delta_{3}(m, y(m))\right] \mathrm{d} m
\end{aligned}
$$

for all $t \geq \tau$. The definition (7) then gives

$$
\begin{aligned}
& x_{r}(t-\tau)=e^{-H \tau} x_{r}(t)+e^{-H \tau} L y(t)-L y(t-\tau) \\
& -\int_{t-\tau}^{t} e^{H(t-m-\tau)}\left[K y(m)+\delta_{3}(m, y(m))\right] \mathrm{d} m
\end{aligned}
$$

for all $t \geq \tau$. By subtracting (12) from (10) and using the definition of $M_{\tau}$ in (6), it follows that

$$
\begin{aligned}
& M_{\tau} x_{r}(t)=e^{-H \tau} L y(t)-L y(t-\tau) \\
& -\int_{t-\tau}^{t} e^{H(t-m-\tau)}\left[K y(m)+\delta_{3}(m, y(m))\right] \mathrm{d} m \\
& +\int_{t-\tau}^{t} e^{A_{2}(t-m-\tau)} \delta_{2}(m, y(m)) \mathrm{d} m
\end{aligned}
$$

for all $t \geq \tau$. Using the invertibility of $M_{\tau}$, we conclude that

$$
x_{r}(t)=\hat{x}_{r}(t)
$$

holds for all $t \geq \tau$, where

$$
\begin{aligned}
& \hat{x}_{r}(t)=M_{\tau}^{-1}\left[e^{-H \tau} L y(t)-L y(t-\tau)\right] \\
& -M_{\tau}^{-1} \int_{t-\tau}^{t} e^{H(t-m-\tau)}\left[K y(m)+\delta_{2}(m, y(m))\right. \\
& \left.\quad+L \delta_{1}(m, y(m))\right] \mathrm{d} m \\
& +M_{\tau}^{-1} \int_{t-\tau}^{t} e^{A_{2}(t-m-\tau)} \delta_{2}(m, y(m)) \mathrm{d} m
\end{aligned}
$$

Hence, when $\delta_{1}$ and $\delta_{2}$ are known, (15) provides the exact value of $x_{r}(t)$ for all $t \geq \tau$.

\section{Main Result For Time-VARYing Systems}

\section{A. Statement of Main Result and Remarks}

This section shows how the finite time observer method from the previous section generalizes to time-varying nonlinear systems of the form

$$
\left\{\begin{array}{r}
\dot{z}(t)=A_{1}(t) x_{r}(t)+\delta_{1}(t, z(t)) \\
\dot{x}_{r}(t)=A_{2}(t) x_{r}(t)+\delta_{2}(t, z(t))
\end{array}\right.
$$

where $x_{r}$ is valued in $\mathbb{R}^{n-p}$, $z$ is valued in $\mathbb{R}^{p}$, the output is

$$
y(t)=z(t)+\epsilon(t)
$$

where the functions $A_{i}$ for $i=1$ and 2 are piecewise continuous and bounded, $\epsilon(t)$ is piecewise continuous and bounded by a constant $\bar{\epsilon} \geq 0$, and the functions $\delta_{1}$ and $\delta_{2}$ are piecewise continuous with respect to $t$ and globally Lipschitz with respect to $z$ and such that there is a nonnegative valued continuous function $\bar{\delta}$ for which

$$
\left|\delta_{1}(t, z)\right|+\left|\delta_{2}(t, z)\right| \leq \bar{\delta}(|z|)
$$

holds for all $t \geq 0$ and $z \in \mathbb{R}^{p}$.

Remark 1: The particular structure of (16) does not overly restrict the class of linear systems to which our methods apply because, as noted in the previous section, any dynamics of the form

$$
\dot{X}=A(t) X+\mathcal{F}(t, Y)
$$

having an output $Y=C X$ with $C$ of full rank can be transformed (using a linear time-invariant change of coordinates) into a new system having the form (16). The $\epsilon(t)$ term in (17) represents a measurement disturbance, which is of interest because in practice, measurements are frequently affected by perturbations.

We assume:

Assumption 1: There are a constant $\tau>0$ and a bounded matrix valued function $L$ of class $C^{1}$ with a bounded first derivative such that with the choice $H(t)=A_{2}(t)+$ $L(t) A_{1}(t)$, the matrix

$$
\Lambda(t)=\Phi_{A_{2}}(t, t-\tau)-\Phi_{H}(t, t-\tau)
$$

is invertible for all $t \in \mathbb{R}$.

See below for methods to check Assumption 1. We next define

$$
\begin{aligned}
& \delta_{3}(t, z)=L(t) \delta_{1}(t, z)+\delta_{2}(t, z) \text { and } \\
& \delta_{4}(t, z)=-\left[D(t) z+\delta_{3}(t, z)\right], \text { where } \\
& D(t)=\dot{L}(t)-H(t) L(t) .
\end{aligned}
$$

We also set

$$
\begin{aligned}
& \hat{x}_{r}(t)= \\
& \Lambda(t)^{-1} \int_{t-\tau}^{t}\left[\Phi_{A_{2}}(m, t-\tau) \delta_{2}(m, y(m)-\epsilon(m))\right. \\
& \left.+\Phi_{H}(m, t-\tau) \delta_{4}(m, y(m)-\epsilon(m))\right] \mathrm{d} m \\
& +\Lambda(t)^{-1}\left[\Phi_{H}(t, t-\tau) L(t)(y(t)-\epsilon(t))\right. \\
& -L(t-\tau)(y(t-\tau)-\epsilon(t-\tau))]
\end{aligned}
$$


for all $t \geq 0$, where $\Lambda(t)^{-1}$ denotes the inverse of $\Lambda(t)$ for each $t$ and similarly for other inverses. We prove:

Theorem 1: If (16) satisfies Assumption 1, then

$$
x_{r}(t)=\hat{x}_{r}(t)
$$

holds for all $t \geq \tau$.

Remark 2: One can more easily check Assumption 1 when $n-p=1$ because in that case, $\Phi_{A_{2}}$ and $\Phi_{H}$ take the one dimensional forms

$\Phi_{A_{2}}\left(t, t_{0}\right)=e^{-\int_{t_{0}}^{t} A_{2}(m) \mathrm{d} m}$ and $\Phi_{H}\left(t, t_{0}\right)=e^{-\int_{t_{0}}^{t} H(m) \mathrm{d} m}$

If $n-p>1$, then checking Assumption 1 may be more difficult because then the formulas for $\Phi_{A_{2}}$ and $\Phi_{H}$ would be harder to compute. On the other hand, it is easier to find explicit formulas for $\Phi_{A_{2}}$ and $\Phi_{H}$ than for $\Phi_{A}$ where $A$ is the function in (19) because the dimension of $A$ is larger than the dimensions of $A_{2}$ and $H$. This is an advantage of the reduced order approach over full order observers.

Remark 3: If there exist two constants $\tau>0$ and $\varpi \in$ $(0,1)$ and a function $L$ such that $\mid \Phi_{A_{2}}(t, t-\tau)^{-1} \Phi_{H}(t, t-$ $\tau) \mid \leq \varpi$ for all $t \geq 0$, then Assumption 1 will hold and $\Lambda^{-1}$ is bounded. To see why, note that in this case, $I-\Phi_{A_{2}}(t, t-$ $\tau)^{-1} \Phi_{H}(t, t-\tau)$ is invertible for all $t \geq 0$ and

$$
\begin{aligned}
& {\left[I-\Phi_{A_{2}}(t, t-\tau)^{-1} \Phi_{H}(t, t-\tau)\right]^{-1}=} \\
& \sum_{k=0}^{\infty}\left[\Phi_{A_{2}}(t, t-\tau)^{-1} \Phi_{H}(t, t-\tau)\right]^{k}
\end{aligned}
$$

holds for all $t \geq 0$. Therefore,

$$
\left|\left[I-\Phi_{A_{2}}(t, t-\tau)^{-1} \Phi_{H}(t, t-\tau)\right]^{-1}\right| \leq \frac{1}{1-\varpi}
$$

holds for all $t \geq 0$, by the geometric sum formula. It follows that $\Lambda(t)^{-1}$ exists for all $t \geq 0$ and

$$
\begin{aligned}
& \left|\Lambda(t)^{-1}\right|= \\
& \left|\left(I-\Phi_{A_{2}}(t, t-\tau)^{-1} \Phi_{H}(t, t-\tau)\right)^{-1} \Phi_{A_{2}}(t, t-\tau)^{-1}\right| \\
& \leq \frac{\left|\Phi_{A_{2}}(t, t-\tau)^{-1}\right|}{1-\varpi}
\end{aligned}
$$

which is bounded because $\Phi_{A_{2}}(t, t-\tau)^{-1}$ is bounded (because $A_{2}$ is bounded and because $\Phi_{A_{2}}^{-1}$ is the fundamental solution associated with $A_{2}$ ). Moreover $\Lambda^{-1}$ will be bounded (when the inverse exists for all $t$ ) if the system is periodic because then $\Lambda^{-1}$ is continuous and periodic.

Remark 4: If $\epsilon$ is unknown, then the exact estimate (23) cannot be used because (22) contains $\epsilon$. However, we can use (23) to build the approximate observer

$$
\begin{aligned}
& x_{r}^{*}(t)= \\
& \Lambda(t)^{-1} \int_{t-\tau}^{t}\left[\Phi_{A_{2}}(m, t-\tau) \delta_{2}(m, y(m))\right. \\
& \left.+\Phi_{H}(m, t-\tau) \delta_{4}(m, y(m))\right] \mathrm{d} m \\
& +\Lambda(t)^{-1}\left[\Phi_{H}(t, t-\tau) L(t) y(t)-L(t-\tau) y(t-\tau)\right] .
\end{aligned}
$$

Since $A_{2}$ and $H$ are bounded, it follows from Gronwall's inequality that $\sup _{m \in[t-\tau, t]}\left|\Phi_{H}(m, t-\tau)\right|$ and $\sup _{m \in[t-\tau, t]}\left|\Phi_{A_{2}}(m, t-\tau)\right|$ are bounded functions of $t$. Hence, if $\Lambda(t)^{-1}$ is also bounded, then we can find a constant $l_{a}>0$ such that $\left|x_{r}^{*}(t)-x_{r}(t)\right| \leq l_{a}|\epsilon|_{\infty}$ holds for all $t \geq \tau$.

\section{B. Proof of Theorem 1}

For each $t \geq 0$, the variable $x_{v}(t)=\Phi_{A_{2}}(t, 0) x_{r}(t)$ satisfies

$$
\begin{aligned}
\dot{x}_{v}(t)= & -\Phi_{A_{2}}(t, 0) A_{2}(t) x_{r}(t) \\
& +\Phi_{A_{2}}(t, 0)\left[A_{2}(t) x_{r}(t)+\delta_{2}(t, z(t))\right] \\
= & \Phi_{A_{2}}(t, 0) \delta_{2}(t, z(t)) .
\end{aligned}
$$

We now integrate (27) between $t-\tau$ and $t \geq \tau$ to obtain

$$
x_{v}(t)=x_{v}(t-\tau)+\int_{t-\tau}^{t} \Phi_{A_{2}}(m, 0) \delta_{2}(m, z(m)) \mathrm{d} m .
$$

We can easily use the definition of $x_{v}$ to obtain

$$
\begin{aligned}
& \Phi_{A_{2}}(t-\tau, 0)^{-1} \Phi_{A_{2}}(t, 0) x_{r}(t)=x_{r}(t-\tau) \\
& +\int_{t-\tau}^{t} \Phi_{A_{2}}(t-\tau, 0)^{-1} \Phi_{A_{2}}(m, 0) \delta_{2}(m, z(m)) \mathrm{d} m .
\end{aligned}
$$

For each continuous function $\Omega: \mathbb{R} \rightarrow \mathbb{R}^{\ell \times \ell}$, the function $\Psi_{\Omega}\left(t, t_{0}\right)=\Phi_{\Omega}\left(t, t_{0}\right)^{\top}$ satisfies

$$
\frac{\partial \Psi_{\Omega}}{\partial t}\left(t, t_{0}\right)=-\Omega(t)^{\top} \Psi_{\Omega}\left(t, t_{0}\right)
$$

Using the semigroup property of flow maps, we conclude that $\Psi_{\Omega}(t, 0)=\Psi_{\Omega}(t, t-\tau) \Psi_{\Omega}(t-\tau, 0)$ holds for all $t \geq$ $\tau$. Hence, $\Psi_{\Omega}(t, 0)^{\top}=\Psi_{\Omega}(t-\tau, 0)^{\top} \Psi_{\Omega}(t, t-\tau)^{\top}$, and $\Psi_{\Omega}(m, 0)=\Psi_{\Omega}(m, t-\tau) \Psi_{\Omega}(t-\tau, 0)$, which implies that $\Psi_{\Omega}(m, 0)^{\top}=\Psi_{\Omega}(t-\tau, 0)^{\top} \Psi_{\Omega}(m, t-\tau)^{\top}$, for all $m \geq t-\tau$ and $t \geq \tau$. The preceding equalities give $\Phi_{\Omega}(t, 0)=\Phi_{\Omega}(t-$ $\tau, 0) \Phi_{\Omega}(t, t-\tau)$ and $\Phi_{\Omega}(m, 0)=\Phi_{\Omega}(t-\tau, 0) \Phi_{\Omega}(m, t-\tau)$, so we can deduce from (29) that

$$
\begin{aligned}
& \Phi_{A_{2}}(t, t-\tau) x_{r}(t)=x_{r}(t-\tau) \\
& +\int_{t-\tau}^{t} \Phi_{A_{2}}(m, t-\tau) \delta_{2}(m, z(m)) \mathrm{d} m .
\end{aligned}
$$

Moreover, the choice $x_{s}(t)=x_{r}(t)+L(t) z(t)$ satisfies

$$
\begin{aligned}
\dot{x}_{s}(t)= & A_{2}(t) x_{r}(t)+\delta_{2}(t, z(t))+\dot{L}(t) z(t) \\
& +L(t)\left[A_{1}(t) x_{r}(t)+\delta_{1}(t, z(t))\right] \\
= & H(t) x_{r}(t)+\dot{L}(t) z(t)+\delta_{3}(t, z(t)) \\
= & H(t) x_{s}(t)+[\dot{L}(t)-H(t) L(t)] z(t) \\
& +\delta_{3}(t, z(t)) .
\end{aligned}
$$

By viewing $\Phi_{H}$ as the inverse of the fundamental matrix for $\dot{q}=H(t) q$, we can then use variation of parameters to obtain

$$
\begin{aligned}
& \Phi_{H}(t, t-\tau) x_{s}(t)=x_{s}(t-\tau)+ \\
& \int_{t-\tau}^{t} \Phi_{H}(m, t-\tau)\left[D(m) z(m)+\delta_{3}(m, z(m))\right] \mathrm{d} m
\end{aligned}
$$

from (32), where $D$ was defined in (21), for all $t \geq \tau$. We deduce from the definition of $x_{s}$ that

$$
\begin{aligned}
& \Phi_{H}(t, t-\tau)\left[x_{r}(t)+L(t) z(t)\right] \\
& =x_{r}(t-\tau)+L(t-\tau) z(t-\tau) \\
& +\int_{t-\tau}^{t} \Phi_{H}(m, t-\tau)\left[D(m) z(m)+\delta_{3}(m, z(m))\right] \mathrm{d} m,
\end{aligned}
$$

which we can reorganize to obtain

$$
\begin{aligned}
& \Phi_{H}(t, t-\tau) x_{r}(t)=x_{r}(t-\tau) \\
& -\Phi_{H}(t, t-\tau) L(t) z(t)+L(t-\tau) z(t-\tau) \\
& +\int_{t-\tau}^{t} \Phi_{H}(m, t-\tau)\left[D(m) z(m)+\delta_{3}(m, z(m))\right] \mathrm{d} m .
\end{aligned}
$$


We now subtract (35) from (31) to obtain

$$
\begin{aligned}
& \Lambda(t) x_{r}(t)=\int_{t-\tau}^{t} \Phi_{A_{2}}(m, t-\tau) \delta_{2}(m, z(m)) \mathrm{d} m \\
& -\int_{t-\tau}^{t} \Phi_{H}(m, t-\tau)\left[D(m) z(m)+\delta_{3}(m, z(m))\right] \mathrm{d} m \\
& +\Phi_{H}(t, t-\tau) L(t) z(t)-L(t-\tau) z(t-\tau)
\end{aligned}
$$

for all $t \geq \tau$. Since Assumption 2 ensures that $\Lambda(t)$ is invertible for all choices of $t$, the theorem follows.

\section{ILLUSTRATION}

\section{A. The studied problem}

To illustrate Theorem 1, let us consider the following variant of a system from [7, p. 143]:

$$
\left\{\begin{array}{l}
\dot{\xi}_{4}=\xi_{3} v_{1} \\
\dot{\xi}_{3}=\xi_{2} v_{1} \\
\dot{\xi}_{2}=v_{2} \\
\dot{\xi}_{1}=v_{1}
\end{array}\right.
$$

with $\left(\xi_{1}, \xi_{2}, \xi_{3}, \xi_{4}\right)$ valued in $\mathbb{R}^{4}$ and the input $\left(v_{1}, v_{2}\right)$ valued in $\mathbb{R}^{2}$. Then (37) is a nonholonomic system in chained form.

We assume that the variables $\xi_{4}, \xi_{3}$ and $\xi_{1}$ are measured, but that $\xi_{2}$ is not measured. Also, we assume that there is no disturbance in the measurement of $\xi_{3}$. Let us consider the problem of making the system (37) track

$$
\left(\xi_{1 r}(t), \xi_{2 r}(t), \xi_{3 r}(t), \xi_{4 r}(t)\right)=(2 \sin (t), 0,0,0) .
$$

We can recast this as a problem of stabilizing the origin of a time-varying system using the classical time-varying change of variables

$$
x_{1}=\xi_{1}-\xi_{1 r}(t)
$$

and by selecting the feedback

$$
v_{1}\left(t, x_{1}\right)=-2 \operatorname{sign}\left(x_{1}\right) \sqrt{\left|x_{1}\right|}+2 \cos (t)
$$

where $\operatorname{sign}: \mathbb{R} \rightarrow \mathbb{R}$ is the function defined by $\operatorname{sign}(m)=$ $\frac{m}{|m|}$ when $m \neq 0$ and $\operatorname{sign}(0)=0$. They result in

$$
\left\{\begin{array}{l}
\dot{\xi}_{4}=\xi_{3}\left[-2 \operatorname{sign}\left(x_{1}\right) \sqrt{\left|x_{1}\right|}+2 \cos (t)\right] \\
\dot{\xi}_{3}=\xi_{2}\left[-2 \operatorname{sign}\left(x_{1}\right) \sqrt{\left|x_{1}\right|}+2 \cos (t)\right] \\
\dot{\xi}_{2}=v_{2}(t) \\
\dot{x}_{1}=-2 \operatorname{sign}\left(x_{1}\right) \sqrt{\left|x_{1}\right|} .
\end{array}\right.
$$

We require $v_{2}$ to be bounded by 1 , i.e., that $\left|v_{2}(t)\right| \leq 1$ for all $t \geq 0$. As an immediate consequence, it follows that the finite escape time phenomenon does not occur and $\xi_{2}(t)$ is bounded by an affine function of $t$. By integrating the last equation of (41), we deduce that $\sqrt{\left|x_{1}(t)\right|}=\sqrt{\left|x_{1}(0)\right|}-t$ when $t \in\left[0, \sqrt{\left|x_{1}(0)\right|}\right]$ and $x_{1}(t)=0$ for all $t \geq \sqrt{\left|x_{1}(0)\right|}$. Hence, for all $t \geq \sqrt{\left|x_{1}(0)\right|}$, we have $\xi_{2}(t) x_{1}(t)=0$.

\section{B. Observer Design}

Since $\xi_{2}(t) x_{1}(t)=0$ holds for all $t \geq \sqrt{\left|x_{1}(0)\right|}$, and since only $\xi_{2}$ is not measured, our next objective is to construct an observer for the two dimensional system

$$
\left\{\begin{array}{l}
\dot{\xi}_{3}=2 \cos (t) \xi_{2} \\
\dot{\xi}_{2}=v_{2}(t)
\end{array}\right.
$$

for all $t \geq \sqrt{\left|x_{1}(0)\right|}$. With the notation of the previous section, this system can be rewritten as

$$
\left\{\begin{aligned}
\dot{z}(t) & =A_{1}(t) x_{r}(t)+\delta_{1}(t, z(t)) \\
\dot{x}_{r}(t) & =\delta_{2}(t, z(t))
\end{aligned}\right.
$$

with $x_{r}(t)=\xi_{2}(t)$, the output $z(t)=\xi_{3}(t), A_{1}(t)=$ $2 \cos (t), A_{2}(t)=0, \epsilon(t)=0, \delta_{1}(t, z)=0$, and $\delta_{2}(t, z)=$ $v_{2}(t)$. We next check that Theorem 1 applies, with $\tau=2 \pi$ and $y=\xi_{3}$. Choosing $L(t)=-2 \cos (t)$, we obtain $H(t)=$ $A_{2}(t)+L(t) A_{1}(t)=-4 \cos ^{2}(t)$. Hence, since

$$
\Phi_{H}(t, s)=e^{4 \int_{s}^{t} \cos ^{2}(\ell) \mathrm{d} \ell}
$$

and $\Phi_{A_{2}}(t, s)=1$ for all $s \geq 0$ and $t \geq s$, the function $\Lambda$ from (20) in Assumption 1 is $\Lambda(t)=1-e^{4 \pi}$ for all $t \in \mathbb{R}$. We conclude that Assumption 1 is satisfied. Thus Theorem 1 applies, and provides the estimate from (22), namely,

$\hat{x}_{r}(t)=\frac{2 \cos (t)}{1-e^{4 \pi}}\left[-e^{4 \pi} \xi_{3}(t)+\xi_{3}(t-2 \pi)\right]$

$+\frac{1}{1-e^{4 \pi}} \int_{t-2 \pi}^{t}\left(1-e^{4 \int_{t-2 \pi}^{m} \cos ^{2}(\ell) \mathrm{d} \ell}\right) v_{2}(m) \mathrm{d} m$

$+\frac{2}{1-e^{4 \pi}} \int_{t-2 \pi}^{t} e^{4 \int_{t-2 \pi}^{m} \cos ^{2}(\ell) \mathrm{d} \ell}\left(4 \cos ^{3}(m)-\sin (m)\right) \xi_{3}(m) \mathrm{d} m$.

We conclude that

$$
\begin{aligned}
& \xi_{2}(t)=\frac{2 \cos (t)}{1-e^{4 \pi}}\left[-e^{4 \pi} \xi_{3}(t)+\xi_{3}(t-2 \pi)\right] \\
& +\frac{1}{1-e^{4 \pi}} \int_{t-2 \pi}^{t}\left(1-e^{4 \int_{t-2 \pi}^{m} \cos ^{2}(\ell) \mathrm{d} \ell}\right) v_{2}(m) \mathrm{d} m \\
& +\frac{2}{1-e^{4 \pi}} \int_{t-2 \pi}^{t} e^{4 \int_{t-2 \pi}^{m} \cos ^{2}(\ell) \mathrm{d} \ell}\left(4 \cos ^{3}(m)\right. \\
& \quad-\sin (m)) \xi_{3}(m) \mathrm{d} m
\end{aligned}
$$

for all $t \geq \max \left\{2 \pi, \sqrt{\left|x_{1}(0)\right|}\right\}$.

\section{Output feedback tracking}

In this section, we illustrate how (45) can be used to solve a tracking problem that we described in Section IV-A. We design a state feedback for

$$
\left\{\begin{array}{l}
\dot{\xi}_{4}=2 \cos (t) \xi_{3} \\
\dot{\xi}_{3}=2 \cos (t) \xi_{2} \\
\dot{\xi}_{2}=v_{2}(t) .
\end{array}\right.
$$

Let us define

$$
\begin{aligned}
& \zeta_{1}=\xi_{4}-2 \sin (t) \xi_{3}-\cos (2 t) \xi_{2} \\
& \zeta_{2}=\xi_{3}-2 \sin (t) \xi_{2} \\
& \zeta_{3}=\xi_{2} .
\end{aligned}
$$

Using the double angle formula for the sine function, we get

$$
\left\{\begin{array}{l}
\dot{\zeta}_{1}=-\cos (2 t) v_{2}(t) \\
\dot{\zeta}_{2}=-2 \sin (t) v_{2}(t) \\
\dot{\zeta}_{3}=v_{2}(t)
\end{array}\right.
$$

Then the derivative of the positive definite quadratic function $\nu\left(\zeta_{1}, \zeta_{2}, \zeta_{3}\right)=\frac{1}{2}\left[\zeta_{1}^{2}+\zeta_{2}^{2}+\zeta_{3}^{2}\right]$ along all trajectories of (48) is $\dot{\nu}(t)=\left[-\cos (2 t) \zeta_{1}-2 \sin (t) \zeta_{2}+\zeta_{3}\right] v_{2}(t)$. Thus with

$$
v_{2}(t)=-\sigma\left(\left[-\cos (2 t) \zeta_{1}-2 \sin (t) \zeta_{2}+\zeta_{3}\right]\right)
$$

where $\sigma(s)=\frac{s}{\sqrt{1+s^{2}}}$, we obtain

$$
\begin{aligned}
& \dot{\nu}(t)=-\left[-\cos (2 t) \zeta_{1}-2 \sin (t) \zeta_{2}+\zeta_{3}\right] \\
& \times \sigma\left(\left[-\cos (2 t) \zeta_{1}-2 \sin (t) \zeta_{2}+\zeta_{3}\right]\right) .
\end{aligned}
$$


We now use the LaSalle Invariance Principle to check that (48) in closed loop with (49) is globally asymptotically stable to 0 , as follows. Consider any solution $\left(\zeta_{1}(t), \zeta_{2}(t), \zeta_{3}(t)\right)$ of (48)-(49) such that $-\cos (2 t) \zeta_{1}(t)-2 \sin (t) \zeta_{2}(t)+\zeta_{3}(t)=0$ for all $t \geq 0$, Then $v_{2}(t)=0$ and so also $\dot{\zeta}_{i}(t)=0$ for all $t \geq 0$ and $i=1$ to 3 . Consequently,

$$
-\cos (2 t) \zeta_{1}(0)-2 \sin (t) \zeta_{2}(0)+\xi_{3}(0)=0
$$

for all $t \geq 0$. We deduce that $\zeta_{i}(0)=0$ for $i=1$ to 3 (by differentiating through (51) with respect to $t$ and then setting $t=0$ in the result). Consequently $\left(\zeta_{1}(t), \zeta_{2}(t), \zeta_{3}(t)\right)=$ $(0,0,0)$ for all $t \geq 0$. Then, we deduce from the periodic LaSalle Invariance Principle from [14, Theorem 5.26] that (49) renders the origin of (48) globally asymptotically stable.

It follows that the bounded feedback

$$
\begin{aligned}
& v_{2}\left(t, \xi_{2}, \xi_{3}, \xi_{4}\right)=\sigma\left(\cos (2 t)\left(\xi_{4}-2 \sin (t) \xi_{3}-\cos (2 t) \xi_{2}\right)\right. \\
& \left.+2 \sin (t)\left(\xi_{3}-2 \sin (t) \xi_{2}\right)-\xi_{2}\right)
\end{aligned}
$$

renders the origin of (46) globally asymptotically stable. By grouping terms, we obtain

$$
\begin{aligned}
& v_{2}\left(t, \xi_{2}, \xi_{3}, \xi_{4}\right)=\sigma\left(\cos (2 t) \xi_{4}+2[1-\cos (2 t)] \sin (t) \xi_{3}\right. \\
& \left.-\left[\cos ^{2}(2 t)+4 \sin ^{2}(t)+1\right] \xi_{2}\right)
\end{aligned}
$$

which we can combine with (45) to obtain the globally asymptotically stabilizing output feedback

$$
\begin{aligned}
& v_{2}(t)=\sigma\left(\cos (2 t) \xi_{4}+2[1-\cos (2 t)] \sin (t) \xi_{3}\right. \\
& -\mathcal{L}(t) \int_{t-2 \pi}^{t}\left(1-e^{\mathcal{E}(m, t)}\right) v_{2}(m) \mathrm{d} m \\
& -2 \mathcal{L}(t) \int_{t-2 \pi}^{t} e^{\mathcal{E}(m, t)}\left(4 \cos ^{3}(m)-\sin (m)\right) \xi_{3}(m) \mathrm{d} m \\
& \left.-2 \cos (t) \mathcal{L}(t)\left[-e^{4 \pi} \xi_{3}(t)+\xi_{3}(t-2 \pi)\right]\right)
\end{aligned}
$$

where $\mathcal{E}(m, t)=2(m-t+2 \pi)+\sin (2 m)-\sin (2 t)$ and

$$
\mathcal{L}(t)=\frac{\cos ^{2}(2 t)+4 \sin ^{2}(t)+1}{1-e^{4 \pi}} .
$$

Notice that $v_{2}$ is a solution of an implicit equation.

We performed simulations, which show the efficiency of our approach. Fig. 1 shows the simulation of the closed loop nonlinear time varying system (41) with $v_{2}$ defined in (52). Since our simulation shows good stabilization and tracking, it helps illustrate our general theory, in the special case of the system (37). We choose $x_{1}(0)=-0.5$ which implies that $\sqrt{\left|x_{1}(t)\right|}=1 / \sqrt{2}-t$ when $t \in[0,1 / \sqrt{2}]$ and $x_{1}(t)=0$ for all $t \geq 1 / \sqrt{2}$. This is evident from the simulation as well.

\section{CONCLUSIONS}

We proposed a new type of reduced order finite time observers. The observers apply to time-varying systems. We conjecture that it can be used to solve a problem of constructing interval observers that is similar to those in [9]. We plan to apply our observer to solve a dynamic output feedback stabilization problem for a MIMO nonlinear system. We will study other extensions. In particular, we hope to combine the main result of the present paper with the result of [8] and to the case where there are a delay and a disturbance in the input and where the outputs are only available on some finite time intervals.
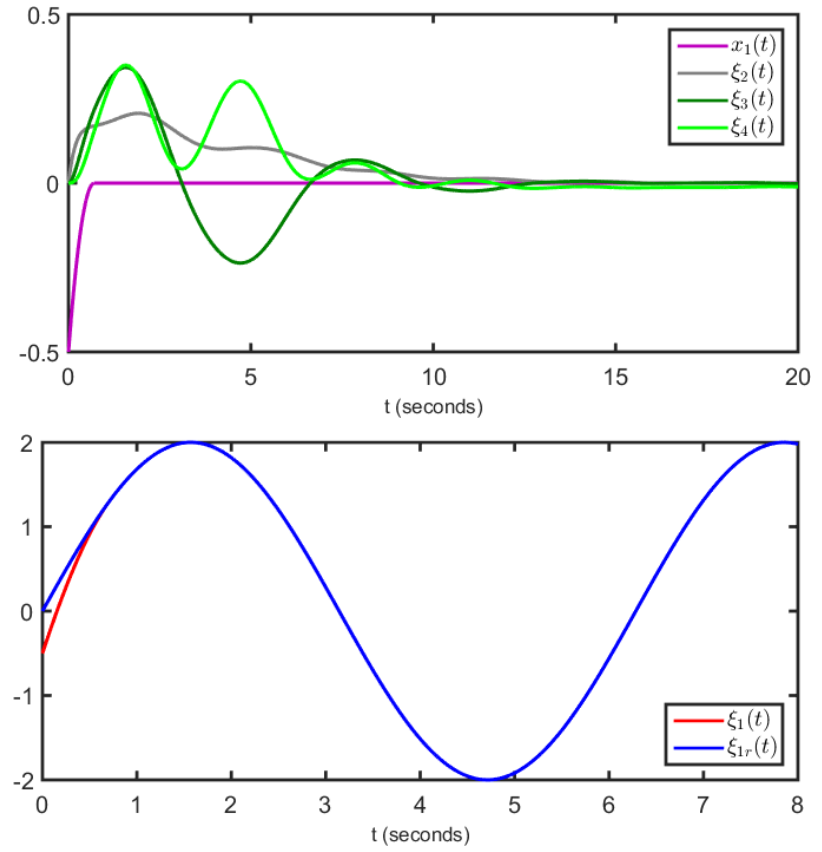

Fig. 1. Simulation results.

\section{REFERENCES}

[1] F. Bonnans and P. Rouchon. Commande et Optimisation de Systèmes Dynamiques. Les Éditions de l'Ecole Polytechnique, Palaiseau, France, 2005.

[2] R. Engel and G. Kreisselmeier. A continuous time observer which converges in finite time. IEEE Transactions on Automatic Control, 47(7):1202-1204, 2002.

[3] F. Friedland. Reduced-order state obervers. In H. Unbehauen, editor, Control Systems, Robotics and Automation - Vol. VIII, pages 26-36. Eoless Publishers Co. Ltd., Oxford, United Kingdom, 2009.

[4] F. Lopez-Ramirez, A. Polyakov, D. Efimov, and W. Perruquetti. Finitetime and fixed-time observer design: Implicit Lyapunov function approach. Automatica, 87:52-60, 2018.

[5] D. Luenberger. Observers for multivariable systems. IEEE Transactions on Automatic Control, 11(2):190-197, 1966.

[6] D. Luenberger. Introduction to Dynamic Systems. John Wiley and Sons, New York, 1979.

[7] M. Malisoff and F. Mazenc. Constructions of Strict Lyapunov Functions. Springer, New York, 2009.

[8] F. Mazenc, S. Ahmed, and M. Malisoff. Finite time estimation through a continuous-discrete observer. International Journal of Robust and Nonlinear Control, to appear, https://doi.org/10.1002/rnc.4286.

[9] F. Mazenc, E. Fridman, and W. Djema. Estimation of solutions of observable nonlinear systems with disturbances. Systems and Control Letters, 79:47-58, 2015.

[10] P. Menold, R. Findeisen, and F. Allgower. Finite time convergent observers for nonlinear systems. In Proceedings of the IEEE Conference on Decision and Control, pages 5673-5678, Maui, HI, 2003.

[11] W. Perruquetti, T. Floquet, and E. Moulay. Finite time observers: application to secure communication. IEEE Transactions on Automatic Control, 53(1):356-360, 2008.

[12] T. Raff and F. Allgower. An impulsive observer that estimates the exact state of a linear continuous time system in predetermined fnite time. In Proceedings of the Mediterranean Conference on Control and Automation, pages 1-3, Athens, Greece, 2007.

[13] T. Raff and F. Allgower. An observer that converges in finite time due to measurement-based state updates. IFAC Proceedings Volumes, 41(2):2693-2695, 2008.

[14] S. Sastry. Nonlinear Systems Analysis, Stability, and Control. Springer, New York, 1999.

[15] F. Sauvage, M. Guay, and D. Dochain. Design of a nonlinear finite time converging observer for a class of nonlinear systems. Journal of Control Science and Engineering, 2007(36954), 2007.

[16] E. Sontag. Mathematical Control Theory, Second Edition. Springer, New York, 1998. 